\title{
THE USE OF UNDERGRADUATE PROJECT COURSES FOR TEACHING IMAGE AND SIGNAL PROCESSING TECHNIQUES AT PURDUE UNIVERSITY
}

\author{
Edward J Delp and Yung-Hsiang Lu \\ School of Electrical and Computer Engineering, \\ Purdue University West Lafayette, Indiana, USA 47907-2035 \\ ace@purdue.edu and yunglu@purdue.edu
}

\begin{abstract}
This paper describes our approaches to introduce service learning and research concepts from image and signal processing into the undergraduate ECE curriculum at Purdue University. In particular, we describe two project courses we have developed: one is in the context of the Purdue Engineering Projects in Community Service (EPICS) program and the other is a new course known as Vertically Integrated Projects (VIP). We will describe three projects: EPICS TCHA, EPICS C-SPAN, and VIP Multimedia. These projects provide students, from first-year students to seniors, with the opportunities to integrate real-world solutions and fundamental materials covered in the classroom.
\end{abstract}

Index Terms - signal processing, image processing, multimedia, community service

\section{INTRODUCTION}

Signal processing education typically starts with a course on signals and systems and a second course on probability and stochastic processes. Even though these theoretical courses provide solid foundation in learning more advanced topics, some students have difficulty in linking mathematics with real applications. To remedy this situation, we have several courses that can be taken by students from all years and through multiple semesters. The purposes of these courses include: (a) Students are given opportunities to learn the applications of signal processing before, during, or after taking theoretical courses. As a result, the students can appreciate more while learning the theory and understand better how to apply the theory. (b) The courses also provide service to our local community and education opportunities for the students to communicate with project partners. (c) Through the interactions with other students in these projects, the students can develop the skills for team work and leadership. Typical team sizes for both EPICS and VIP are about 5-10 students in any semester.

This paper describes two project courses: EPICS (Engineering Projects in Community Service) and VIP (Vertically Integrated Projects). EPICS is a multi-disciplinary course for students from all departments. In EPICS, each project has a project partner from a local non-profit organization, e.g. the Tippecanoe County Historical Association (TCHA) and the C-SPAN Networks archive. Since the EPICS program was created eleven years ago, more than 2000 students have participated [1]. EPICS has received accreditation as a course for ABET senior design credits if a student takes EPICS in the same team for two semesters.

VIP, in contrast, is a research-oriented course. There is no project partner. Instead, the projects are defined by the team advisers. A VIP project is often a research project with a narrower scope or an application of a research project. Hence, the undergraduate students have opportunities working with graduate research assistants. For example, the VIP multimedia project uses concepts from video and image processing research developed in Purdue Video and Image Processing Laboratory. In VIP, the students are also encouraged to publish their results. A conference paper [2] was produced in Summer of 2005 as the result of a VIP project in Spring 2005. Currently, there are 4 VIP teams and 30 students. The VIP class is in the process of obtaining ABET design credits approval for the ECE program.

It should be emphasized that we have used ideas and techniques developed in our research programs for both the EPICS Teams and VIP Team to be described below.

\section{SAMPLE PROJECTS}

\subsection{EPICS-TCHA}

The main goal of of EPICS TCHA Team is to work with the Tippecanoe County Historical Association (TCHA) and help them develop methods and systems for disseminating information about Tippecanoe County; this is the same county in Indiana where Purdue University West Lafayette campus is located. The Team has developed and delivered to TCHA the following features, as can be seen at http://tcha.ecn.purdue.edu/.

- Image Database: The Team has designed and deployed a content-addressable image and video database system. This system can search for images and videos that describe objects in the TCHA museum and events 
held by TCHA. The system uses very sophisticated image and video processing tools to search for images and videos based on their content and whether they are similar to other images a user has found. Many types of search criteria are possible. The images are protected by both visible and invisible watermarks. The system is shown in Figure 1.

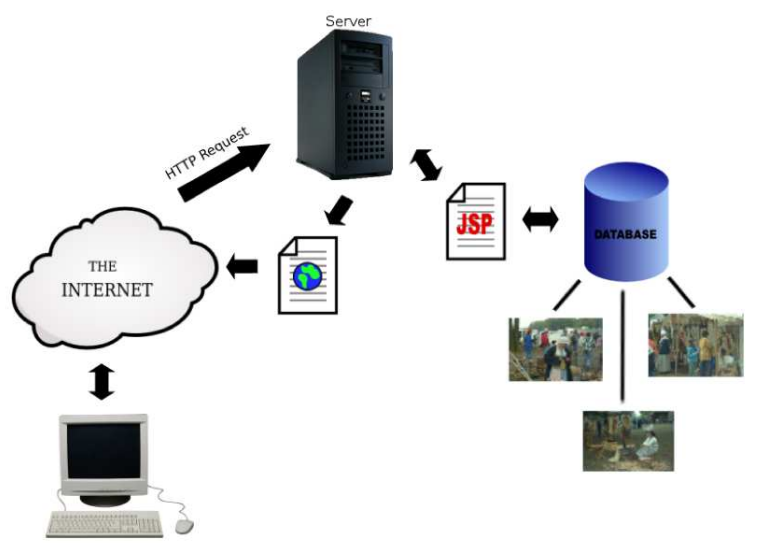

Fig. 1. The TCHA-EPICS Image Database System

- A Day in The Life: The database system will indicate to a user what events have occurred in the the history of the county base on a day or data that the user indicates. The system is based on a free text based MySQL database designed by the Team.

- Oral History: The purpose of this project is to acquire and edit video interviews of Tippecanoe County residents for distribution in multiple formats. The project is composed of four parts: (a) A mobile video system was designed and constructed in order to interview Tippecanoe residents by video using two cameras. (b) After acquiring the video, the video is edited. (c) The video is then converted into MPEG-2 format and incorporated into a DVD. (d) A Quicktime-formatted video is created so that the video can be served for streaming through the Internet. Key frames are identified and extracted and entered in the database of images and videos described above.

- Virtual Tour: This is a multimedia virtual tour of the Fowler House Museum. The tour allows web users to move virtually throughout the museum and examine many of the museum displays and exhibits.

The above projects are multi-year efforts and have resulted in relatively large systems being deployed by the students. They need to understand concepts of documenting their work so that the next semester's team, which may include many new members, can continue the work. The students also need to understand how large systems need to be maintained and supported.

\subsection{EPICS-CSPAN}

The goal of the EPICS C-SPAN Team is to provide a publicly accessible video database containing local government or community agency meetings. Currently no archival records exist in a format that is easy to use, searchable, and freely available to the public on the web. The Team is building such a database and takes the same approach as currently implemented by the C-SPAN cable television channels: all videos will be completely unedited. The Team is sponsored and advised by the C-SPAN Archives, located near the Purdue Campus. The system as currently deployed is available at http://cspan1.ecn.purdue.edu/ and the architecture is shown in Figure 2. The Team is working with four local government or government-like groups: the Lafayette City Council, the West Lafayette City Council, the Tippecanoe County Council, and the Purdue University Board of Trustees.

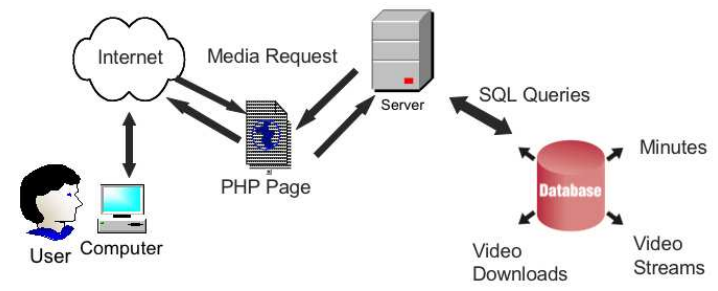

Fig. 2. The CSPAN-EPICS Database System

The system is based on an MySQL database system with a web front end. At this time the system mainly consists of searchable minutes of the meetings held by the above groups. Several videos of meetings held by the groups are also in the database. Indexing of videos is planned for Fall 2006.

\subsection{VIP-Multimedia}

The VIP course is similar to EPICS in that a student may take VIP for multiple semesters. The main difference is that VIP topics are research-oriented and there is no community partner. VIP started as a project-based course for wireless communication in 2002. As a result, all existing projects are related to wireless communication. There are four projects currently: (a) multimedia, video, and image for hand held devices, (b) wireless for transportation by detecting road conditions and transmitting the data through a wireless network, (c) e-stadium providing instant replay of a game on a PDA, and (d) wave propagation for indoor wireless communication. We are the co-advisers for the VIP Multimedia Team.

The Multimedia Team has investigated several topics about providing multimedia on handheld devices, such as a PDA. 


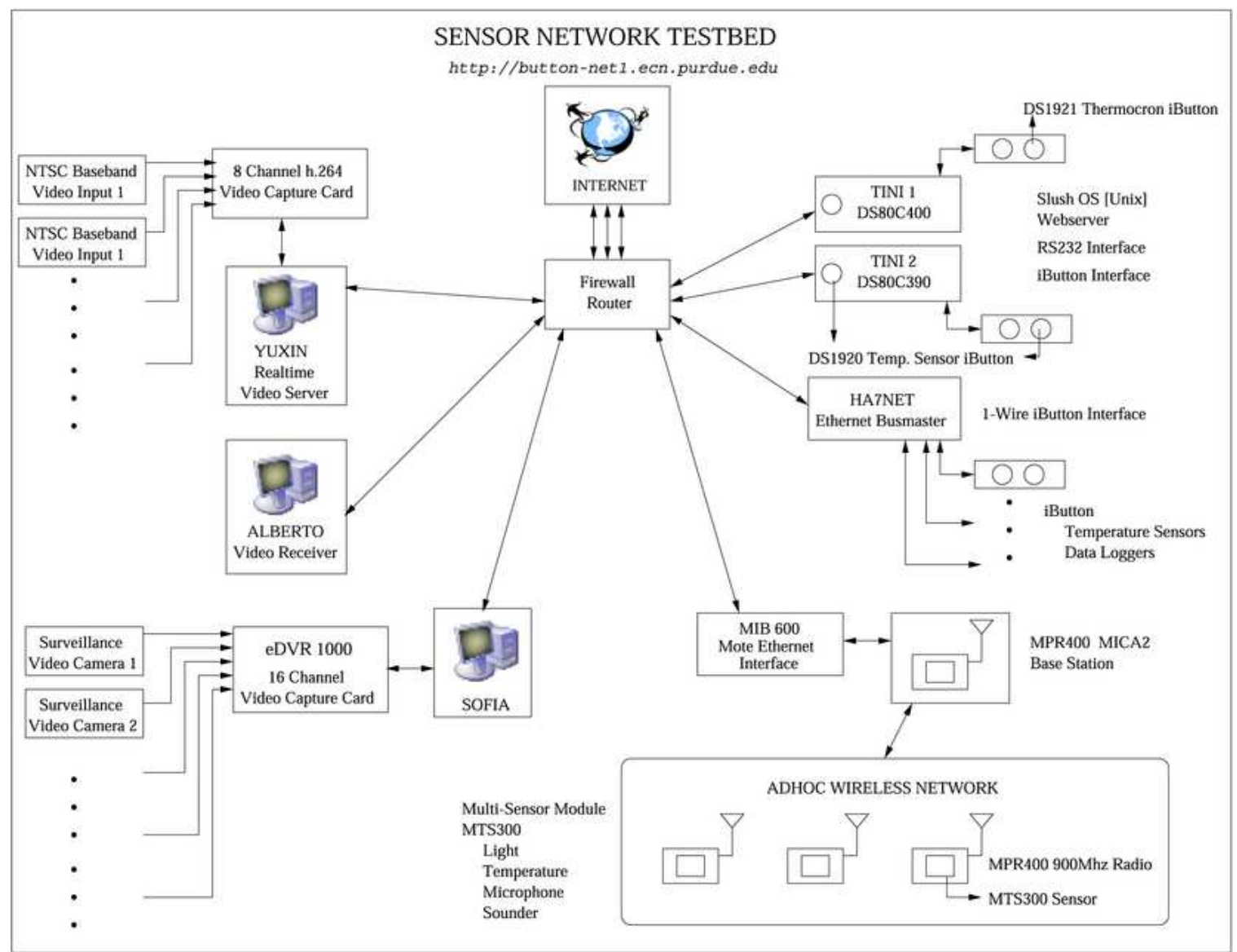

Fig. 3. The integrated sensor system.

During 2003-2005, the team studied how to use images, in addition to maps, for assisting navigation [2]. Since 2005, the team has been integrating the information from multiple sources to monitor the environment for security and safety. The information is collected by video cameras and multiple sensors (temperature, light, and audio). The purpose of this project includes (a) Use a subset of sensors to trigger the other sensors. For example, temperature and light sensors are used to turn on the video cameras because video cameras require significantly more resources in bandwidth, storage, and power consumption. Hence, cameras are used only when certain events have been detected. (b) Perform analytics on the collected data to identify danger or threats by detecting suspicious behavior of a person. The team has successfully ported and tested the software for the sensors and the video cameras separately and is in the process of integrating them. Figure 3 shows the overall system with multiple data sources of sensors and videos.

\section{ASSESSMENT}

Through these projects, the students can understand how to apply textbook knowledge to real-world problems. The students can also learn the state-of-the-art research issues that are being investigated at Purdue University. Moreover, the same projects continue even though different students join and leave every semester. Thus, the students learn how to organize projects despite the changes in personnel as this is typical in most companies. Because these courses are different from traditional lecture courses, the students' accomplishments are assessed through a different set of metrics. Discussion, presentation, and documentation are emphasized in these project courses.

The EPICS program has done many formal assessments of the students in the program [1]. It has been shown that EPICS provides a very positive and lasting experience for the students. The VIP program is new and we are beginning to formalize the assessment of the program. 


\section{CONCLUSION}

We are confident that the EPICS and VIP teams have been able to enhance their understanding of signal and image processing concepts and methods by the types of projects we are doing. The students are learning about working on teams and thinking as engineers, instead of typical undergraduate students that only worry about homework in the coming week. Finally, they are also getting a "taste" of what research is about and how concepts from the classroom can be extended into practical problems.

\section{REFERENCES}

[1] Edward J Coyle, Leah H. Jamieson, and William C. Oakes, "Integrated Engineering Education and Community Service: Themes for the Future of Engineering Education," Journal of Engineering Education, pp. 7-11, January 2006.

[2] Shantanu Gautam, Gabi Sarkis, Edwin Tjandreanegara, Evan Zelkowitz, Yung-Hsiang Lu, and Edward J Delp, "Multimedia for Mobile Users: Image Enhanced Navigation," in Multimedia Content Analysis, Management, and Retrieval, IS\&T/SPIE Symposium on Electronic Imaging, 2006. 